and emptied pancreas the majority of these lipochondria are single structures, in the emptied pancreas a much greater number are found which are compound bodies composed of a number of vesicles. 'The size of the lipochondria ranges from $\frac{1}{2} \mu$ to $3 \mu$, the majority being $1 \frac{1}{2} \mu$ in diameter. In the liver the lipoid droplets are not vesiculated in life.

Both the liver lipoid droplets and the lipochondria of the pancreas are selectively stained in vivo by methylene blue, toluidene blue, neutral red, brilliant cresyl blue and nile blue sulphate. In hypertonic solutions of sodium chloride, the methylene blue and toluidene blue stained bodies of both organs undergo changes of form (myelination) similar to those which phospholipide droplets undergo in forming myelin figures. The stained lipochondria also become myelinated vigorously in sodium chloride solutions as low as 1.4 per cent, which is far below the concentration isotonic for the pancreas ${ }^{8}$. Myelination of the methylene blue stained bodies has also been obtained in solutions of sodium bicarbonate, calcium chloride and slightly in sucrose solutions. The myelin figures so formed closely simulate the classical Golgi net. Palade and Claude ${ }^{4}$ showed that the lipoid droplets of the liver became myelinated in classical Golgi fixatives and could be stabilized and impregnated with osmic acid to give the classical Golgi apparatus of the liver. Their observation that they also become myelinated after treatment with alcoholic solutions of sudan black to simulate the classical Golgi apparatus has been confirmed.

The neutral red stained droplets of both liver and pancreas do not show myelin figure formation in hypertonic solutions of sodium chloride.

In distilled water, irrespective of the presence or absence of dye in the water, the methylene blue, toluidene blue, and neutral red stained lipoid bodies of both liver and pancreas readily destain and form artefact structures, the 'vacuome-crinome' complexes. These are composed of a clear nonrefractile vacuole, within and at one pole of which there is segregated a small amount of refractile yellowish material. No such artefacts form in undamaged material in 0.85 per cent sodium chloride.

There seems to be no doubt that the lipoid bodies of the liver are the Golgi bodies of the live cells, and from the data above there is seen a remarkable parallel between the properties of the vitally stained lipoid droplets of the pancreas and liver, both in the selectiveness of staining, in the simulation of the classical Golgi nets on advanced myelination, and in the formation of vacuome-crinome complexes. These data further confirm the view of Hirsch ${ }^{1}$, Palade and Claude 4 and Baker 7 that the Golgi bodies of the living pancreas are, in fact, the lipochondria. It is established, however, contrary to the opinion of Palade and Claude, that the lipochondria of the pancreas are vesiculated in life. Also, it is established that the methylene blue stained droplets of the liver are not neo-formations or bodies other than the lipoid droplets, but that, in fact, they are the lipoid droplets.

In discussing the relation of the lipochondria. or Golgi bodies of the pancreas to the formation of zymogen granules, the nomenclature of Hirsch ${ }^{1}$ will be followed in describing the walls of these bodies as the Golgi externa, and the vesicles enclosed by them as the Golgi interna. Hirsch ${ }^{1,5}$ and Ries ${ }^{2}$ both depict, as the earliest prozymogen granules free of encirclement by Golgi externa, bodies which are approximately the size of mature zymogen granules (that is, $1 \frac{1}{2} \mu$ ). A study of the empty and restituting mouse pancreases obtained by feeding or pilocarpine injections shows that this is incorrect. The earliest. prozymogen visible in live tissue is less than $\frac{1}{2} \mu$ in diameter and is, in fact, almost at the limits of microscopic visibility. These prozymogen granules are well spaced from each other, and, when the objective is focused slightly beyond them, they are readily visible as dull darkish bodies surrounded by clear 'vacuoles' at the centres of which they are situated. However, on bringing them into proper focus, they are seen as glistening yellowish granules which are, in fact, not surrounded by vacuoles. This young prozymogen does not stain with the vital dyes listed above, nor are the individual granules surrounded oven partially by chromophilic or osmiophilic material. The chromophile and osmiophile lipochondria or Golgi bodies are found at the base of the prozymogen mass. The interna of these are larger than the young prozymogen. No evidence was found for the view that the prozymogen formed within the Golgi interna, and it was definitely disproved that they matured within the interna. Nor was there any evidence that the smaller non-vesiculated Golgi bodies were a stage in a possible transformation to prozymogen granules, since they stained more readily with vital dyes than did the larger lipochondria, and were quite as osmiophilic as them. It has been concluded that the vesiculated lipochondria are not organelles of zymogen synthesis, but are very elementary myelin forms, and that their further myelination after vital staining is due to alterations in their surface properties.

This work was carried out in the Zoological Department, University of Melbourne.

$$
\begin{gathered}
\text { Plant Virus Research Unit, } \\
\text { Molteno Institute, } \\
\text { Cambridge. } \\
\text { Feb. 5. }
\end{gathered}
$$

N. Xeros

1 Hirsch, H. C., "Form- und Stoffiwechsel der Golgi-Korper" (Berlin: Borntraeger, 1939).

${ }^{2}$ Ries, E., Z. Zellforsch., 22, 523 (1935).

' Worley, L. G., J. Morph., 75, 261 (1944).

- Palade, G. E., and Claude, A., J. Morph., 85, 35 and 71 (1949).

${ }^{5}$ Hirsch, H. C., Z. Zellforsch., 15, 36 (1932).

- Opie, E. L., J. Exp. Med., 89, 185 (1949).

? Baker, J. R., Quart. J. Micr. Sci., 90, 293 (1949).

\section{Record of a Whale Shark off the West Coast of Malaya}

IN going through some records which have survived the Japanese occupation, a photograph and account of the catching of a specimen of the rare whale shark, Rhineodon typicus, have come to light.

The fish was caught on November 25, 1940, off a sandy beach on the coast of Pangkor Island off the west coast of the Malay peninsula. Unfortunately there is no record of the sex. The fish was thirty-five feet long and twenty-three feet between the outstretched pectoral fins. It was caught in a beach seine about one mile in length, operated by Madras fishermen who migrate to Malaya. The fish was cut up and thrown back into the sea. The fishermen weighed just over thirteen thousand three hundred pounds of flesh and then tired.

$$
\text { D. W. Le Mare }
$$

(Director)

H.Q. Fisheries Department,

Federation of Malaya and Singapore, Penang.

Nov. 6. 